\title{
Tryptophan metabolite activation of the aryl hydrocarbon receptor regulates IL-10 receptor expression on intestinal epithelia
}

\author{
JM Lanis ${ }^{1,2}$, EE Alexeev ${ }^{1,2}$, VF Curtis ${ }^{1,2}$, DA Kitzenberg ${ }^{1,2}$, DJ Kao ${ }^{1,2}$, KD Battista ${ }^{1,2}$, ME Gerich $^{1,2}$, \\ LE Glover ${ }^{1,2}$, DJ Kominsky ${ }^{1,3,4}$ and SP Colgan ${ }^{1,2,4}$
}

IL-10 is a potent anti-inflammatory cytokine that inhibits the production of proinflammatory mediators. Signaling by IL-10 occurs through the IL-10 receptor (IL-10R), which is expressed in numerous cell types, including intestinal epithelial cells (IECs), where it is associated with development and maintenance of barrier function. Guided by an unbiased metabolomics screen, we identified tryptophan (Trp) metabolism as a major modifying pathway in interferon- $\gamma$ (IFN $\gamma$ )-dominant murine colitis. In parallel, we demonstrated that IFN $\gamma$ induction of indoleamine 2,3-dioxygenase 1, an enzyme that catalyzes the conversion of Trp to kynurenine (Kyn), induces IL-10R1 expression. Based on these findings, we hypothesized that IL-10R1 expression on IEC is regulated by Trp metabolites. Analysis of the promoter region of IL-10R1 revealed a functional aryl hydrocarbon response element, which is induced by Kyn in luciferase-based IL-10R1 promoter assays. Additionally, this analysis confirmed that IL-10R1 protein levels were increased in response to Kyn in IEC in vitro. Studies using in vitro wounding assays revealed that Kyn accelerates IL-10-dependent wound closure. Finally, reduction of murine dextran sodium sulfate colitis through Kyn administration correlates with colonic IL-10R1 expression. Taken together, these results provide evidence on the importance of IL-10 signaling in intestinal epithelia and implicate AHR in the regulation of IL-10R1 expression in the colon.

\section{INTRODUCTION}

A complex interdependency exists within the human intestine, where trillions of microbes interact with the host mucosa. While most of the microbiota benefit host health, pathogens and dysbiosis can lead to disease. ${ }^{1-5}$ It is for this reason that a selective barrier is essential to intestinal homeostasis. Epithelial barrier is maintained through multiple levels of cell-cell interaction that functionally seals the epithelial monolayer. Defects in barrier function have been linked to many human diseases, most notably inflammatory bowel disease. ${ }^{6}$

The importance of IL-10 signaling is well established in models of inflammatory bowel disease. Indeed, mice lacking IL-10 or the IL-10 receptor (IL-10R) are predisposed to spontaneous colitis. ${ }^{711}$ Functional IL-10 signaling in the epithelium promotes cell proliferation and barrier formation, ${ }^{12,13}$ and results in homeostasis and maintenance of the epithelia. ${ }^{13}$ It was recently demonstrated that activation of the epithelial IFN $\gamma$ receptor, which is well established to induce barrier dysfunction, ${ }^{14,15}$ also induces the expression of the IL-10R1 in both cultured IEC and mice. ${ }^{13}$ These results provide a compelling example of how balanced inflammatory processes also elicit inflammatory resolution.

One of the major signaling cascades induced by activation of IFN $\gamma$ receptors is induction of tryptophan (Trp) metabolism through the induction of indoleamine 2,3-dioxygenase 1 (IDO1) ${ }^{16,17}$ IDO1 is an enzyme responsible for conversion of Trp to kynurenine $(\mathrm{Kyn})^{18}$ and has been shown to suppress T-cell proliferation through the localized catabolism of Trp. ${ }^{19}$ Expression of IDO1 in intestinal epithelial cells (IECs) strongly correlates with anti-inflammatory properties of dendritic

${ }^{1}$ Mucosal Inflammation Program, Aurora, Colarado, USA. ${ }^{2}$ Department of Medicine, University of Colorado Anschutz Medical Campus, Aurora, Colarado, USA and ${ }^{3}$ Department of Microbiology and Immunology, Montana State University, Bozeman, Montana, USA. Correspondence: DJ Kominsky (douglas.kominsky@montana.edu) or SP Colgan (sean.colgan@ucdenver.edu)

${ }^{4}$ Co-senior authors.

Received 27 May 2016; accepted 15 November 2016; published online 18 January 2017. doi:10.1038/mi.2016.133 
cells, ${ }^{20-24}$ and reduces severity of colitis in mice. ${ }^{23}$ Epithelial IDO1 has also been shown to activate $\beta$-catenin signaling and promote colonic tumorigenesis in inflammation-associated cancer. ${ }^{25}$

Kyn is a Trp metabolite that functions as an endogenous ligand of the aryl hydrocarbon receptor (AHR) transcription factor. $^{26,27}$ This transcription factor has been historically studied in the context of the synthetic dioxin-based compound TCDD (2,3,7,8-tetrachlorodibenzo- $p$-dioxin), and is known to control genes such as $C y p 1 A 1$ that are important for protecting cells from intoxication by various planar aromatic hydrocarbons. ${ }^{28,29}$ More recently, numerous endogenous ligands for AHR have been identified. ${ }^{30}$ For example, Kyn competes with TCDD for binding cytosolic AHR and endogenously regulate systemic inflammation and tolerance. ${ }^{27}$ Additionally, AHR has been implicated in various immune functions, including T-helper type 17 (Th17) development and the expression of IL-17 and IL-22. ${ }^{31-35}$ Taken together, these data lead us to hypothesize that IFN $\gamma$ induces the expression of IL-10R1 through an increase in tissue Trp metabolism and activation of AHR.

\section{RESULTS}

\section{Altered tissue metabolism in mice with DSS colitis}

There is significant current interest in defining metabolomic changes associated with disease progression and inflammatory resolution. ${ }^{36}$ Here, we performed a comprehensive, unbiased screen of metabolites of serum and colonic tissue from active dextran sodium sulfate (DSS) colitis. Mice were exposed to DSS via drinking water and serum and colonic epithelium were collected on day 7 . Figure 1 shows a summary of these results. This data set comprised the analysis of 502 biochemicals of known identity. Principal component analysis revealed that metabolism was significantly altered both locally and systemically in mice administered DSS (Figure 1a). In total, 42 metabolites were found to be significantly increased in the colon, whereas 120 metabolites were present in higher amounts in the serum after DSS exposure. Most notable were changes in several metabolites along the Trp metabolism pathway in both serum and colons of colitic mice (Figure 1b). Trp is metabolized along at least three separate pathways involving various enzymatic signaling cascades and mechanisms (Figure 1c). Catabolites of Trp were changed fundamentally in DSS colitis. In the colon, 5-hydroxyindoleacetate was significantly lower $(P<0.05)$, whereas serotonin and C-glycosyl Trp were increased $(P<0.05$, Figure 1b). The most notable change was increased Kyn levels across both colon $(4.4 \pm 0.39$-fold increase, $P<0.01$, Supplementary Figure $S 1$ online) and serum samples $(2.1 \pm 0.55$ increase, $P<0.01$, Figure $\mathbf{1 b})$ from DSS colitic mice.

\section{Elevated Kyn levels are associated with an upregulation of ID01}

In an attempt to better understand the temporal regulation of Kyn, independent analyses of Kyn levels in mice during various stages of DSS colitis were performed using electrochemical coulometric array detection (EC-HPLC). Over the course of 7 days after DSS, Kyn levels increased in both the colon and serum compared with untreated mice (Figure 2a,c). Kyn levels in the colon and serum gradually increased to over sevenfold the levels found at baseline with increasing time of exposure to DSS, supporting our liquid chromatography-mass spectrometry profiling screen (Figure $\mathbf{2 b , d}$ ). Interestingly, by day $\mathbf{1 0}$, Kyn levels in the colon and serum trend toward normal, suggesting that Kyn may serve as a sensitive biomarker of inflammation and resolution (Figure 2b,d).

Once Trp is internalized, intracellular enzymes become the rate-limiting step in the catabolism of Trp to Kyn and other downstream metabolites. Consistent with previous studies in other cell types, ${ }^{37}$ analysis of our previously published microarray using T84 IEC ${ }^{38}$ (GEO accession number GSE33880: http://www.ncbi.nlm.nih.gov/geo/query/acc.cgi?acc=GSE33880) revealed that the Trp-catabolizing enzyme IDO1 is highly upregulated upon exposure to IFN $\gamma$ (Supplementary Figure S1A), a cytokine that we have previously shown to be temporally upregulated in DSS colitis. ${ }^{13}$ By contrast, other Trp-catabolizing enzymes (e.g., TPH1 and TDO2) remain unchanged with IFN $\gamma$ treatment (Supplementary Figure S1A). In line with the data from IEC exposed to IFN $\gamma$, mice had an increase in colonic IDO1 protein expression over a course of 10 days of DSS and recovery as measured by western blot densitometry (Figure 2e). IDO1 expression and subsequent Kyn production take place in a number of cell types including immune cells; therefore, we investigated whether isolated colonic epithelial cells upregulated IDO1 during DSS. DSS exposure lead to a twofold induction of ido1 transcript levels in whole colon tissue, approximately a threefold induction from colonic mucosal scrapings, and over a 10 -fold induction of ido1 transcript was detected in colonic epithelial cells enriched by immunomagnetic separation (Figure 2f).

The primary known function of IDO1 is the conversion of Trp to Kyn. Therefore, we examined whether IFN $\gamma$ exposure increased Kyn levels in cultured IECs. As shown in Supplementary Figure S1B, this analysis revealed that T84 cell exposure to IFN $\gamma$ resulted in a significant increase in secreted Kyn that can be inhibited by the Trp analog 1-methylDL-Trp (1-MT). IFN $\gamma$ has been shown to control the expression of IL-10R1 on IECs, thus we also analyzed the expression of IL10R1 by quantitative PCR (qPCR) in the presence of IFN $\gamma$ and 1 -MT. While IFN $\gamma$ results in over a 50 -fold increase in il10r1 transcript, the presence of 1-MT skews the expression levels back toward baseline (Supplementary Figure S1C). We also measured transcript levels of a known AHR target, Cyp1A1, in the presence of 1-MT. Interestingly, without IDO1 Trp catabolism, Cyp1A1 levels return to baseline (Supplementary Figure S1C).

To further demonstrate the association between intestinal inflammation and elevated Kyn levels, we determined the levels of Kyn, IL-10R1, and IDO1 in TNF(NF(ARE). ${ }^{39}$ These mice develop a Crohn's disease-like pathology, and also show an increase in systemic Kyn, in line with data from the DSS-treated mice (Supplementary Figure S2A). In turn, TNF(NF(ARE) 

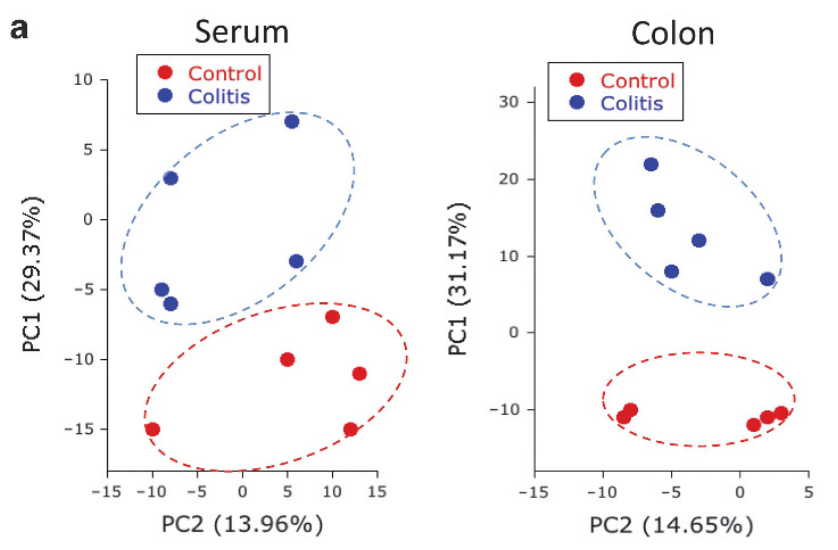

b

\section{Serum}

Colon

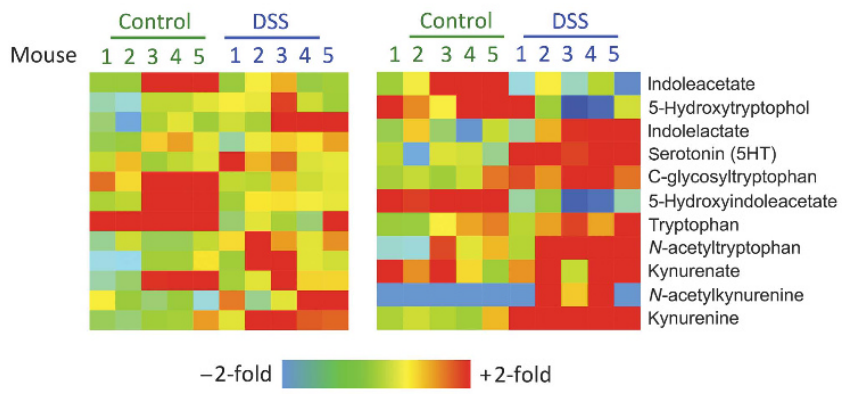

C

IDO1

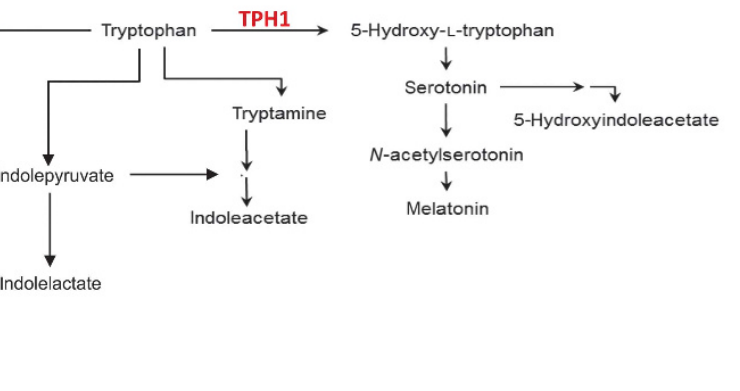

Figure 1 Metabolomic profiling of DSS colitic mice. (a) Principal component analysis of total metabolic differences in control mice and mice receiving $3 \%$ DSS for 7 days. Metabolites were measured by Metabolon using LC/MS and GC/MS analysis, $n=5$. (b) Heat map of metabolites in the Trp pathway that are altered during DSS. (c) Summary of Trp metabolism pathway including the enzymes involved in the primary metabolism of Trp to Kyn or 5-hydroxytryptophan. DSS, dextran sodium sulfate; GC/MS, gas chromatography-mass spectrometry; IDO1, indoleamine 2,3-dioxygenase 1; LC/MS, liquid chromatography-mass spectrometry; PC1, proprotein convertase 1; TPH1, tryptophan hydroxylase 1; Trp, tryptophan.

mice also have an increase in bido1 and il10r1 mRNA in the colonic epithelia (Supplementary Figure S2B). Taken together, these results implicate a significant shift in Trp metabolism toward Kyn mediated by IFN $\gamma$, and provide a link between IFN $\gamma$ signaling and the AHR pathway.

\section{Expression of IL-10R1 on IEC is controlled by AHR ligands} Given the strong association between IL-10R1 and IFN $\gamma^{13}$ and the above-described link between IFN $\gamma$ and IDO1, we investigated the existence of a relationship between Trp metabolism and IL-10R1 regulation in IEC. Kyn, along with other Trp metabolites, functions as an endogenous ligand for the AHR), a transcription factor that responds to planar aromatic hydrocarbons. ${ }^{27,40,41}$ As shown in Figure 3, exposure of T84 IEC to Kyn, the UV-induced Trp derivative FICZ (6formylindolo(3,2-b)carbazole), and the AHR ligand TCDD resulted in a significant increase in both il10r1 and cyp1a1 mRNA levels (all $P<0.05$, Figure 3a). Analysis of the sequence flanking the IL-10R1 gene identified several putative AHR response elements $\left(5^{\prime}\right.$ - T/GNGCGTGA/CG/CA- $\left.3^{\prime}\right)$ within the promoter region. ${ }^{42}$ This $1.4 \mathrm{~kb}$ fragment of the promoter region was cloned upstream of a luciferase reporter, transfected into Caco-2 IEC, exposed to Kyn, FICZ, and IFN $\gamma$ and luciferase activity was measured after 12 h. As shown in Figure 3b, FICZ, Kyn, and IFN $\gamma$ all significantly increased promoter activity compared with vehicle control $(P<0.001)$, suggesting that
Trp metabolites induce IL-10R1 through a mechanism involving AHR.

To further determine the influence of AHR on IL-10R1 expression, we used short hairpin RNA knockdown (KD) (short hairpin RNA (shRNA)) to individually deplete AHR and the AHR nuclear transporter (ARNT) in Caco-2 and T84 IEC, respectively (Supplementary Figure S3A,B). Caco-2 AHR-KD cells exposed to Kyn or FICZ resulted in no significant induction of IL-10R1 compared with cells transfected with a non-targeting control shRNA (Figure 3d,e). Similarly, T84 ARNT-KD cells revealed no detectable levels of IL-10R1 at baseline and no induction with FICZ, Kyn, or TCDD (Figure 3f,g).

Expression of IL-10R1 on IEC occurs predominantly on the apical surface of the cell. ${ }^{13}$ To determine whether AHR ligand-induced AHR activation results in IL-10R1 surface expression in adherent cells, we performed cell surface ELISA on Caco-2 shRNA control, ARNT-KD cell lines, and Caco-2 cells treated with the pharmacological AHR inhibitor $\mathrm{CH} 223191$. In line with our western blot results, the expression of IL-10R1 increased $8.96 \pm 2.06$-fold $(P<0.01)$ with FICZ, $4.38 \pm 1.06$-fold $(P<0.05)$ with Kyn, and $6.26 \pm$ 1.36 -fold $(P<0.01)$ with TCDD in shRNA control cells (Figure 3c). No increase in IL-10R1 was detectable with FICZ, Kyn, or TCDD exposure of ARNT-KD or AHR inhibitor-treated cells $(P=$ not significant, Figure $3 c)$, 
a

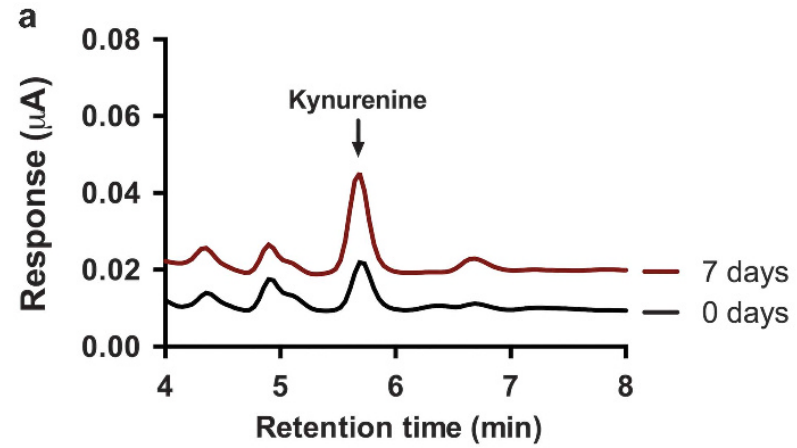

c

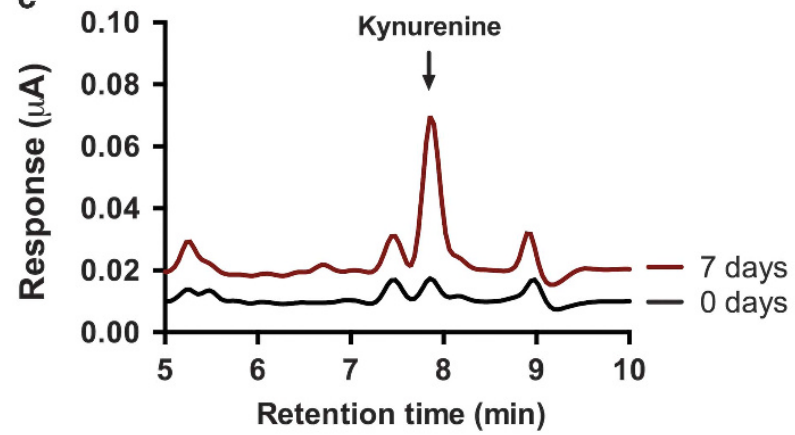

e

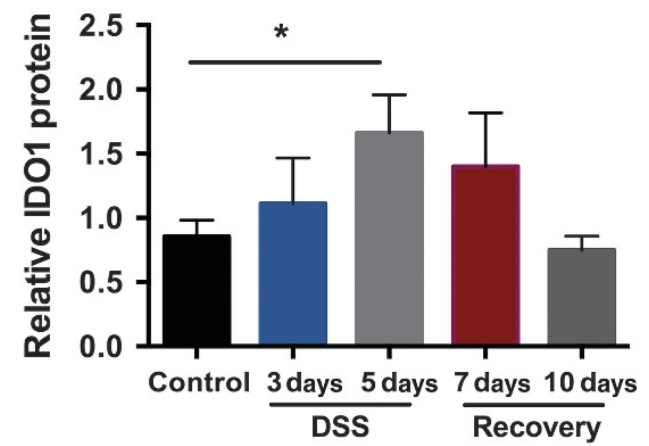

b

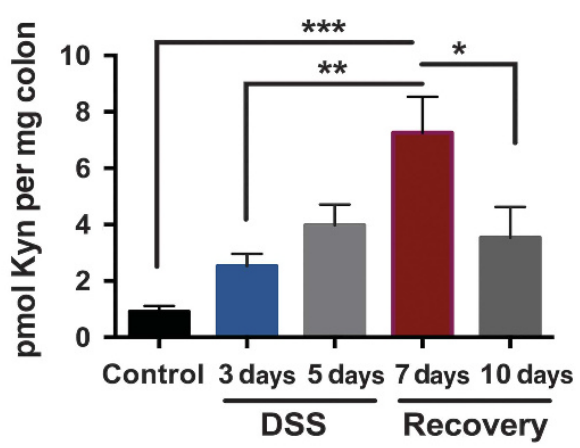

d
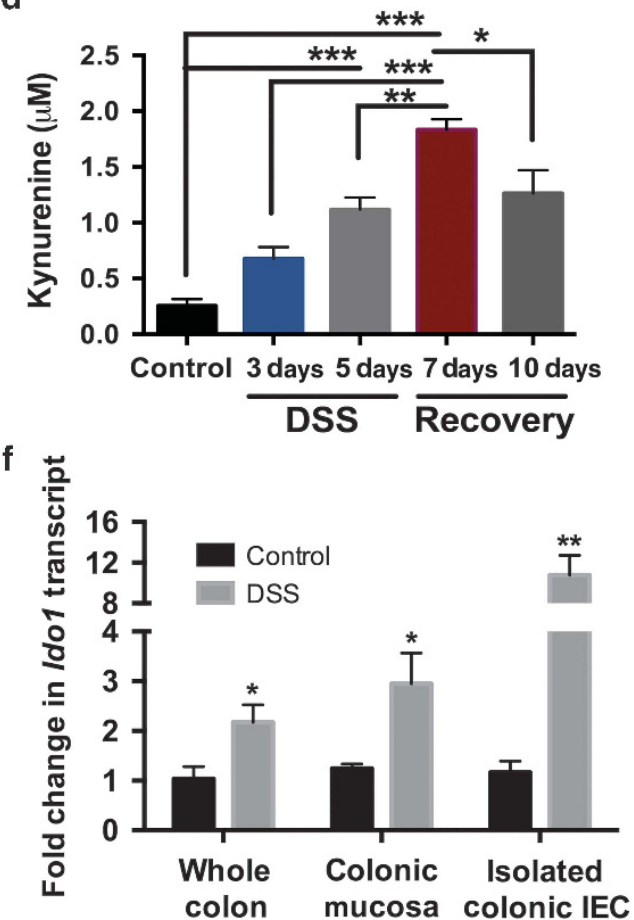

Figure 2 Kynurenine profiling during active inflammation. (a) EC-HPLC analysis of Kyn in the colon of DSS mice at days 0 and 7, $n=5$. (b) Concentration of Kyn in the colon of mice receiving $3 \%$ DSS for up to 5 days, $n=5\left({ }^{\star} P<0.05\right.$, ${ }^{* *} P<0.01$, $\left.{ }^{* * *} P<0.001\right)$. (c) EC-HPLC analysis of Kyn in the serum of DSS mice at days 0 and $7, n=5$. (d) Concentration of Kyn in the serum of mice receiving $3 \%$ DSS for up to 5 days, $n=5$ ( ${ }^{\star} P<0.05$, ${ }^{* *} P<0.01$, $\left.{ }^{* * *} P<0.001\right)$. (e) Western blot quantification of IDO1 levels in the colons of mice receiving $3 \%$ DSS for up to 5 days, $n=5$ ( $\left.{ }^{\star} P<0.05\right)$. (f) qPCR of $i d o 1$ transcript levels in whole colon, colonic mucosal scrapings, and enriched colonic epithelial cells after 6 days of $2.5 \% \mathrm{DSS}, n=5\left({ }^{*} P<0.05 ;{ }^{* *} P<0.01\right)$. DSS, dextran sodium sulfate; EC-HPLC, electrochemical coulometric array detection; IDO1, indoleamine 2,3-dioxygenase 1; Kyn, kynurenine; qPCR, quantitative PCR.

further confirming a role for AHR/ARNT in the regulation of IL-10R1 by Trp metabolites.

Previous work has shown a protective role for FICZ in murine models of colitis. ${ }^{32,43}$ We sought to determine how administration of FICZ in vivo might influence IL-10R1 on the colonic mucosa. Villin-Cre + conditional ARNT-knockout (KO) mice along with Cre - littermates were gavaged with $50 \mu \mathrm{g} \mathrm{kg}^{-1}$ of FICZ, and mRNA levels of IL-10R1 in the colonic epithelia were analyzed after 6 and $12 \mathrm{~h}$. The illor $1 \mathrm{mRNA}$ levels were twofold higher (Supplementary Figure S4A) and the IL-10R1 protein levels trended higher (Supplementary Figure S4B,C) in the FICZ-treated control mice at 6 and $12 \mathrm{~h}$, respectively. Meanwhile, the ARNT-KO mice showed no increase in illorl transcript or protein expression
(Supplementary Figure S4). By $12 \mathrm{~h}$ of FICZ exposure, il10r1 levels in the Cre - mice returned to control levels, with no detectable change in ARNT-KO tissue (Supplementary Figure S4). These results provide a compelling in vivo correlate for regulation of IEC IL-10R1 by ARNT.

\section{Activation of AHR promotes epithelial wound healing}

We next determined whether AHR-dependent IL-10R1 was functional. Our previous work demonstrated that IL-10 signaling is important in IEC tight junction formation and ultimately barrier function; therefore, we performed an in vitro scratch wound assay using T84 IEC and followed the gap closure over $72 \mathrm{~h}$ in the presence of a timed combination of Kyn followed by recombinant human IL-10. As depicted in 

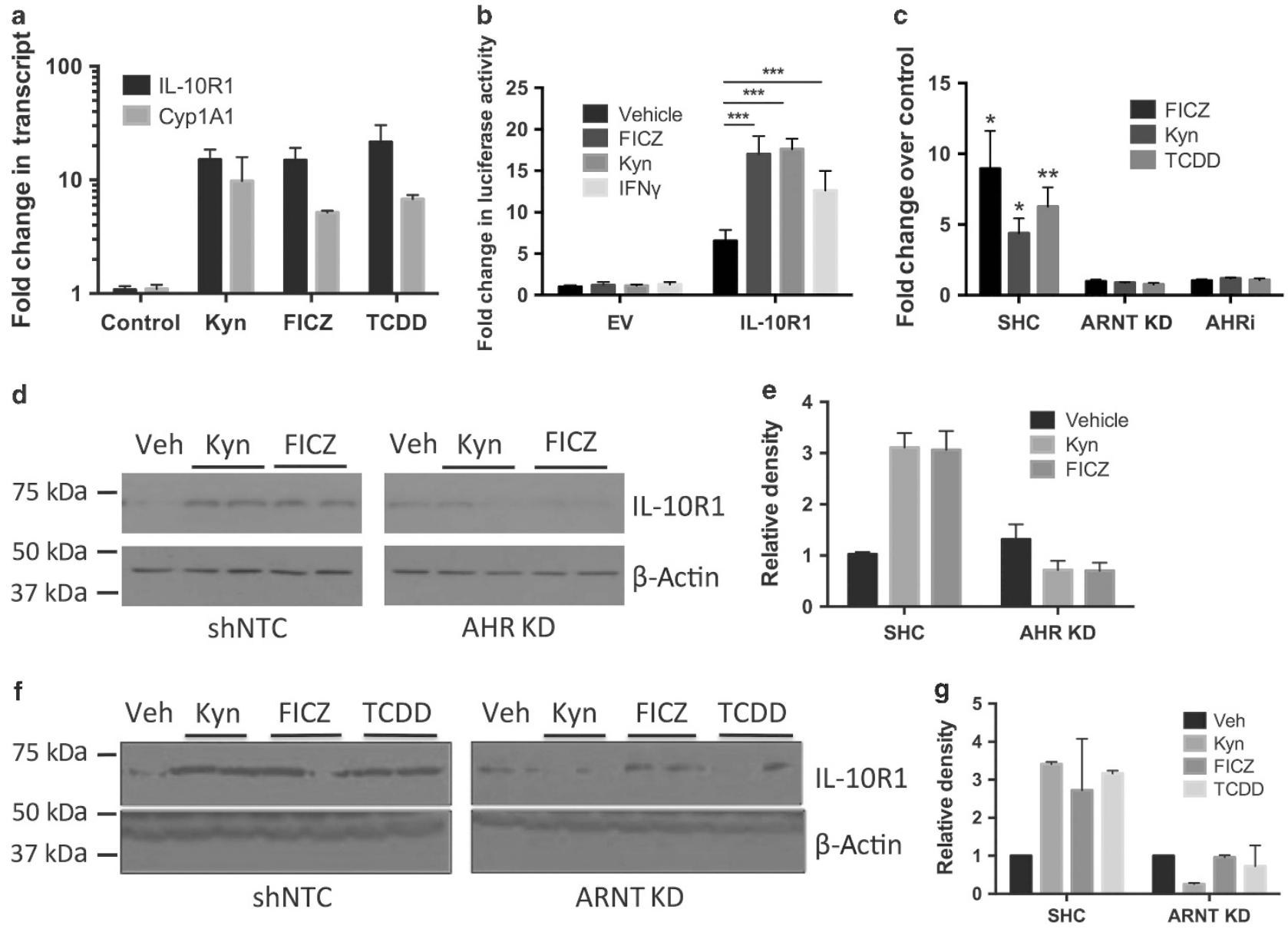

Figure 3 IL-10R1 expression in response to AHR ligands. (a) qPCR of il10r1 and cyp1a1 transcript levels in T84 IEC in response to AHR ligands. Confluent monolayers of T84 cells were treated with Kyn $(100 \mu \mathrm{m})$, FICZ $(250 \mathrm{~nm})$, or TCDD $(30 \mathrm{~nm})$ for 6 h. Error bars represent the s.e.m. of five replicates. (b) Luciferase-based assay of IL-10R1 promoter activity in response to AHR ligands FICZ and Kyn. IFN $\gamma$ served as a positive control. Caco-2 IECs were transfected with pGL3 containing the IL-10R1 promoter region or empty pGL3 vector as control, treated for $12 \mathrm{~h}$ with $100 \mu \mathrm{M} \mathrm{Kyn,} 250 \mu \mathrm{m} \mathrm{FICZ,}$ or $10 \mathrm{ng} \mathrm{ml}^{-1}$ IFN $\gamma$ and luciferase activity measured, $\left.n=3{ }^{* \star \star} P<0.001\right)$. (c) Cell surface ELISA of IL-10R1 expression in shControl, shRNA ARNT KD, and AHR inhibitor-treated Caco-2 IEC. Confluent monolayers of Caco-2 IEC were treated with $100 \mu \mathrm{m} \mathrm{Kyn,} 250 \mathrm{~nm}$ FICZ, or $30 \mathrm{~nm}$ TCDD for $24 \mathrm{~h}$. Error bars represent the s.e.m. of triplicate samples $\left({ }^{\star} P<0.05 ;{ }^{* *} P<0.01\right)$. (d) Western blot analysis of IL-10R1 levels in shRNA AHR KD Caco-2 IEC. Confluent monolayers of Caco-2 cells containing a non-template control (shNTC) or shRNA specific for AHR were treated with FICZ or Kyn for $24 \mathrm{~h}$. (e) Densitometry of the western blot analysis in Figure 3d. Relative density of the IL-10R1 bands is normalized to $\beta$-actin. (f) Western blot analysis of IL-10R1 levels in shRNA ARNT KD T84 IEC. Confluent monolayers of T84 cells containing a non-template control (shNTC) or shRNA specific for ARNT were treated with Kyn, FICZ, or TCDD for $24 \mathrm{~h}$. (g) Densitometry of the western blot analysis in Figure 3F. Relative density of the IL-10R1 bands is normalized to $\beta$-actin. AHRi, aryl hydrocarbon response element inhibitor; ARNT, AHR nuclear transporter; ELISA, enzyme-linked immunosorbent assay; EV, empty vector; FICZ, 6-formylindolo(3,2-b)carbazole; IEC, intestinal epithelial cell; IFN $\gamma$, interferon- $\gamma$; IL-10R1, interleukin-10 receptor 1; KD, knockdown IL-10R, interleukin-10 receptor; Kyn, kynurenine; qPCR, quantitative PCR; SHC, short hairpin control; TCDD, 2,3,7,8-tetrachlorodibenzo-p-dioxin; Veh, vehicle.

Figure 4a,b, scratch wounds closed by $82.7 \pm 4.0 \%$ over a $48 \mathrm{~h}$ period. The addition of Kyn alone resulted in a similar response to control monolayers $(87.9 \pm 4.1 \%, P=$ not significant compared with control), whereas the addition of IL-10 alone increased scratch wound closure to $93.6 \pm 3.2 \%$ $(P<0.05$ compared with control). The combination of Kyn and IL-10 lead to $73.0 \pm 4.2 \%$ restitution by $24 \mathrm{~h}(P<0.001$ compared with IL-10 alone) and complete wound closure within $48 \mathrm{~h}$, indicating that Kyn-dependent signaling via IL-10 functionally promotes epithelial wound closure independent of enhanced proliferation (Supplementary Figure S5A).

By stark contrast, shRNA IL-10R1-KD T84 cells exhibited delayed monolayer restitution in response to a combination of
Kyn and IL-10 (Figure 4a-c). After $72 \mathrm{~h}$, the mock-treated control cells closed by only $54.9 \pm 7.4 \%$, and the restitution with Kyn alone was not significant at $47.5 \pm 3.7 \%$ (Figure $\mathbf{4 b}$ ). The addition of IL-10 alone did not promote epithelial wound closure $(63.5 \pm 6.7 \%)$ and the combination of Kyn and IL-10 was not significantly different than mock-treated control cells $(66.4 \pm 4.6 \%)$. Similarly, another AHR ligand, FICZ, also promotes wound healing in T84 IEC in the presence of IL-10 (Figure 5a-c). However, ARNT-KD IECs were unable to recover from the scratch wound regardless of FICZ or IL-10 administration (Figure 5b,c), indicating that the AHR/ARNT pathways appear to be essential for wound closure. To understand how the AHR ligands Kyn and FICZ are 
a

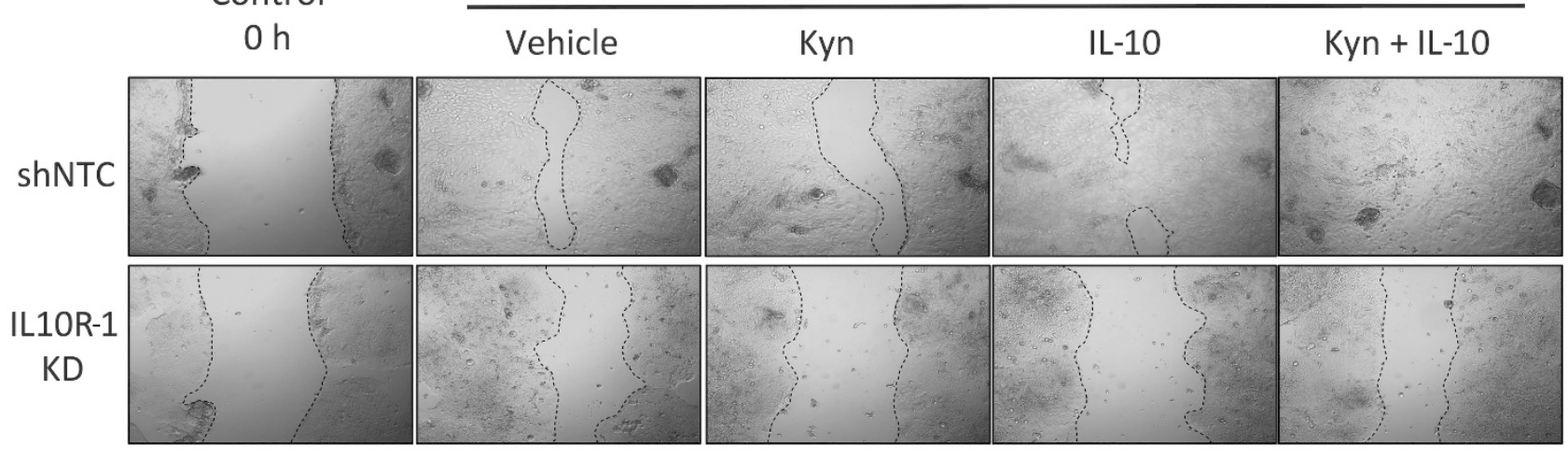

b

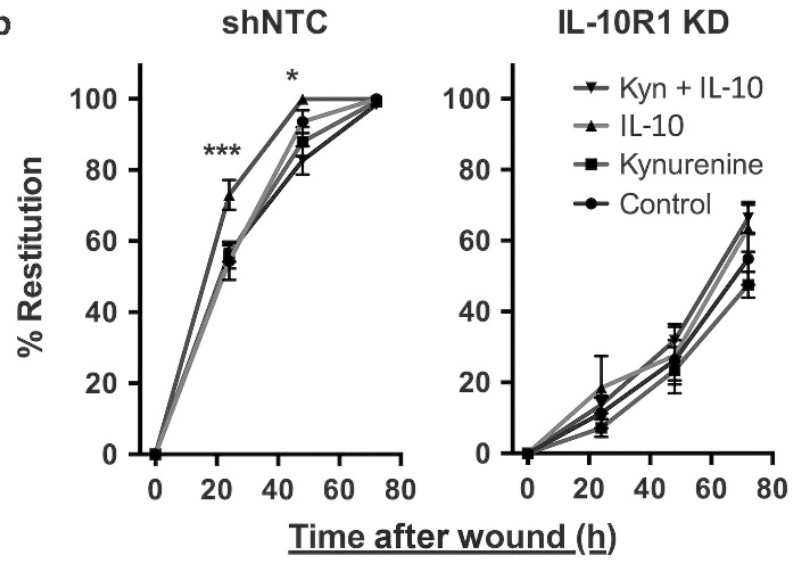

C

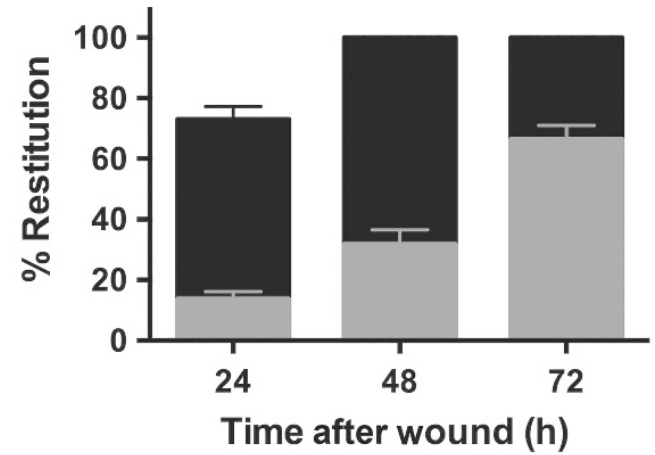

Figure 4 In vitro scratch wound assay in T84 IL-10R1 KD IEC. (a) Representative photos of T84 IEC 0 and $48 \mathrm{~h}$ after Scratch. T84 shNTC (top) and IL-10R1 KD (bottom) were treated with Kyn, IL-10, or both, after wounding and monitored for $48 \mathrm{~h}$. (b) Restitution of scratch wounds (y axis) in T84 shNTC (left) and T84 IL-10R1 KD (right) at 0, 24, 48, and $72 \mathrm{~h}$ (x axis) with control, Kyn, and IL-10 treatment, $n=5$. (c) Comparison of shNTC (black) and IL-10R1 $\mathrm{KD}$ (gray) wound healing with FICZ and IL-10. Error bars represent the s.e.m. of triplicate measurements in five independent experiments $\left({ }^{\star \star \star} P<0.001\right.$ for combination of IL-10 and Kyn compared with IL-10 alone). IL-10R, interleukin-10 receptor; KD, knockdown; Kyn, kynurenine; shNTC, short hairpin non-template control.

promoting wound healing, we next investigated whether the AHR ligands influenced the expression of genes responsible for IEC junction proteins. T84 cells treated with Kyn or FICZ alone had no increase in any of the junctional genes assayed, indicating that the observed wound healing is dependent on an increase in IL-10 signaling and related downstream pathways (Supplementary Figure S5B). In turn, the lack of IL-10R1 or ARNT abrogates the protective influences of the AHR-IL-10 axis in IEC.

\section{Exogenous Kyn protects mice from DSS colitis}

AHR ligands such as FICZ have been shown to be protective in murine models of colitis through multiple mechanisms of immune cell modulation. ${ }^{32,43}$ Our data have shown that the AHR ligand Kyn is increased during inflammation through an upregulation of IDO1 and that this leads to IL-10R expression on intestinal epithelia. This increase in IL-10R1 is associated with enhanced wound healing in vitro; therefore, we next explored the influence of Kyn supplementation in a murine model of DSS colitis. Eight-week-old C57B6 mice were exposed to $2.5 \%$ DSS in the drinking water for 6 days, and then recovered with tap water for 2 additional days. As shown in Figure 6a,b, mice receiving $\mathrm{Kyn}\left(10 \mathrm{mg} \mathrm{kg}^{-1}\right.$ intraperitoneally) lose less weight over 8 days, and had a substantial decrease in disease activity index. Despite the $10 \mathrm{mg} \mathrm{kg}^{-1}$ dose of Kyn, the colonic and systemic levels of Kyn were measured to be no higher than the heightened physiologic levels seen during inflammation in Figure 2 (Supplementary Figure S5C,D). In line with the in vitro wound healing, DSS mice receiving Kyn display a significant reduction in intestinal permeability as measured by FITC-dextran (Figure 6c). This protection in Kyn-treated mice is extended to colon length (Figure 6d,e). Colons in mice receiving DSS alone average $4.3 \pm 0.1 \mathrm{~cm}$, while colon length in Kyn-treated DSS mice averaged $4.8 \pm 0.1 \mathrm{~cm}$ $(P<0.05)$ (Figure 6d,e). Histological analysis of the colon tissue revealed severe inflammation and loss of crypt architecture in DSS mice that is ameliorated with the addition of Kyn (Figure 6f,g). These results are associated with increased IL-10R1 expression in isolated colonic epithelial cells from mice receiving Kyn $(P<0.05)$ (Supplementary Figure S6D).

In contrast, villin-cre + conditional AHR-knockout mice do not respond to Kyn during DSS colitis. As shown in 
a

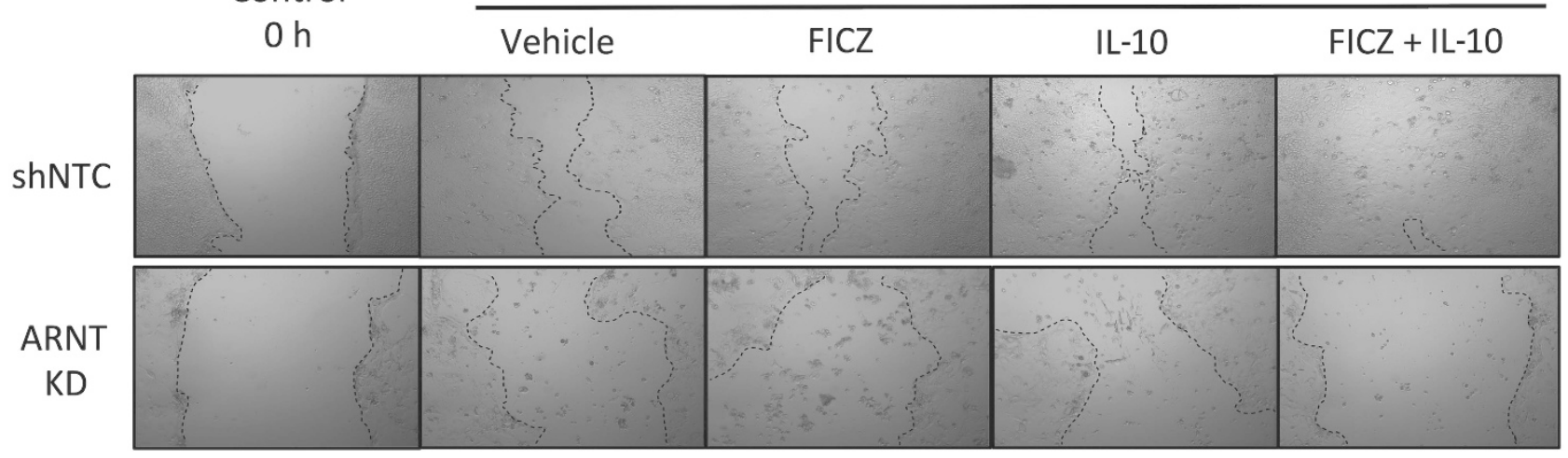

b

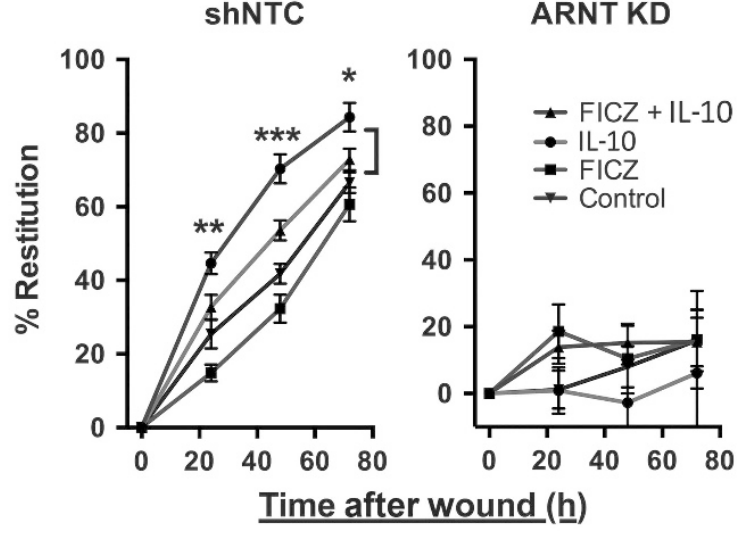

c

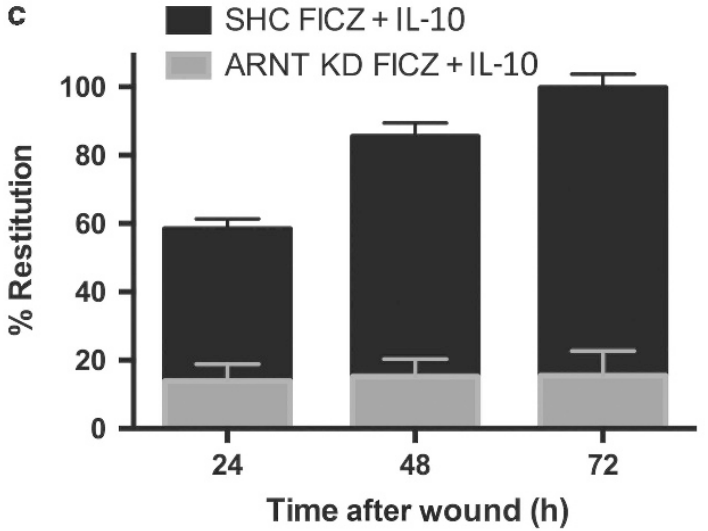

Figure 5 In vitro scratch wound assay in T84 ARNT KD IEC. (a) Representative photos of T84 IEC 0 and $72 \mathrm{~h}$ after scratch. T84 shNTC (top) and ARNT KD (bottom) were treated with FICZ (blue), IL-10 (gray), or both (red), after wounding and monitored for $72 \mathrm{~h}$. (b) Restitution of scratch wounds (y axis) in T84 shNTC (left) and T84 ARNT KD (right) at 0, 24, 48, and $72 \mathrm{~h}$ (x axis) with control, FICZ, and IL-10 treatment, $n=5$. (c) Comparison of shNTC (black) and ARNT KD (gray) wound healing with FICZ and IL-10. Error bars represent the s.e.m. of triplicate measurements in five independent experiments ${ }^{\star \star *} P<0.0001$ for combination of IL-10 and FICZ compared with IL-10 alone). ARNT, aryl hydrocarbon response element nuclear transporter; FICZ, 6-formylindolo(3,2-b)carbazole; IEC, intestinal epithelial cell; IL, interleukin; KD, knockdown; shNTC, short hairpin non-template control.

Supplementary Figure S6A,C, there is no difference in disease activity or weight loss between DSS-exposed AHR-KO mice with or without Kyn. When compared with WT mice treated with Kyn, the AHR-KO mice with Kyn consistently demonstrated more disease symptoms over the course of DSS and recovery $(P<0.001)$, despite no difference in weight loss (Supplementary Figure S6B,D). In line with the results in Figure 6, exposure to Kyn during DSS extended the colon lengths of Cre - control mice, whereas colons from AHR-KO mice with or without Kyn are equal in length after DSS (Supplementary Figure S6E). Interestingly, colonic epithelial cells isolated from the AHR-KO mice after DSS showed no increase in IL-10R1 expression after Kyn treatment (Supplementary Figure S6F). Taken together, these results provide a mechanism for the protective influence of AHR signaling during colitis, and indicate that increased Kyn promotes barrier function through epithelial IL-10 signaling.

\section{DISCUSSION}

IECs function to both facilitate nutrient and fluid transport and provide the primary barrier to luminal antigens. Selective permeability is mediated by specialized anatomical features, including vectorial membrane transport systems and dynamically regulated intercellular junctions. Recent evidence suggests that during ongoing inflammation, proinflammatory signals (e.g., IFN $\gamma$ ) elicit downstream responses that promote inflammatory resolution (e.g., induction of functional IL-10R). ${ }^{13,38,44,45}$ It is clear that intestinal IL-10 signaling is important for homeostasis and the lack of functional IL-10/ IL-10R results in spontaneous colitis in mice. ${ }^{7-9}$ Expression of the IL-10R on IECs is associated with increased transepithelial resistance and barrier function. ${ }^{13}$ In the current work, we define a role for IFN $\gamma$-induced Trp metabolism in inflammation and identify the AHR as the transcription factor responsible for IL-10R1 expression in intestinal epithelia.

Inflammatory metabolism has become an area of intense interest. ${ }^{38}$ Herein, we used an unbiased analysis to define metabolic shifts in ongoing intestinal inflammation. Initial metabolomic studies from mice with DSS colitis revealed that a number of the metabolites along the Trp metabolism pathway were altered both locally (i.e., colon) and systemically (serum). The elevation of Kyn in serum and colonic tissue during colitis was of particular interest. Although we have not directly analyzed Kyn binding to AHR, others have shown that Kyn is a 

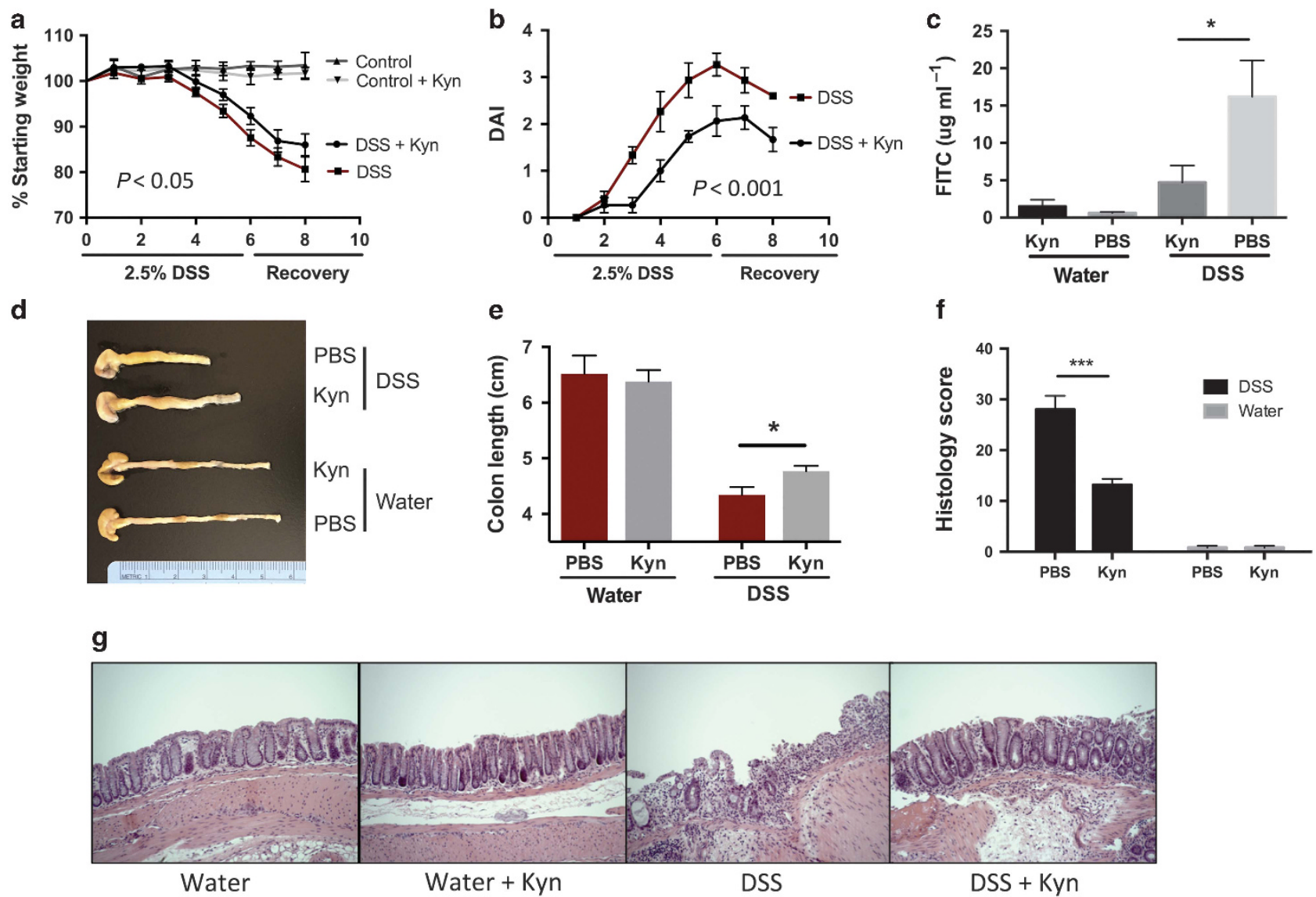

Figure 6 Impact of Kyn supplementation on DSS colitis outcomes. (a) Weight curves during 2.5\% DSS and recovery with and without Kyn administration (10 $\mathrm{mg} \mathrm{kg}^{-1}$ every $48 \mathrm{~h}$ ) $n=5$. (b) Disease activity scores during $2.5 \%$ DSS and recovery with and without Kyn administration (10 mg kg ${ }^{-1}$ every $48 \mathrm{~h}$ ). Disease activity was measured by a combination of weight loss, stool consistency, and bleeding, $n=5$. (c) Intestinal permeability after 6 days of DSS and 2 days of recovery, with and without Kyn. Mice were gavaged with FITC-dextran and permeability was measured by the concentration of FITC (y axis) in the serum, $\left.n=5{ }^{*} P<0.05\right)$. (d) Colon anatomy 8 days after DSS with or without Kyn. (e) Average colon length (y axis) in mice receiving water or DSS with or without Kyn, $n=5\left({ }^{\star} P<0.05\right)$. (f) Histology score after 8 days after DSS with and without Kyn, $n=5$. ( $\left.{ }^{\star * \star} P<0.001\right)$. (g) Representative photos of H\&E-stained colon sections from DSS and control mice with or without Kyn. DSS, dextran sodium sulfate; H\&E, hematoxylin and eosin; Kyn, kynurenine; PBS, phosphate-buffered saline.

demonstrated endogenous ligand for $\mathrm{AHR}^{26,27}$ and can directly compete with TCDD for binding to cytosolic AHR. ${ }^{27}$ Our previous work defined a prominent induction of IFN $\gamma$ in DSS colitis, ${ }^{13}$ and a major IFN $\gamma$ target is IDO1, a widely expressed protein that controls the catabolism of Trp to Kyn.$^{38}$ Increases in Trp metabolism through IDO1 can deplete local Trp levels and inhibit diverse functions, such as T-cell proliferation ${ }^{19}$ and intestinal bacterial growth. ${ }^{46,47}$ Our observations indicate that IFN $\gamma$-induced IDO1 in IEC functions to increase levels of intracellular Kyn. It is notable that other branches of Trp metabolism (e.g., generation of serotonin) were also increased during active colitis (see Figure 1) and implicate a common metabolic process to promote the depletion of local Trp levels. Such metabolic regulation provides an important example of the intricate balance between inflammation and tissue metabolism.

Further investigation into the role of Trp metabolism in IEC revealed that various Trp metabolites induce IEC IL-10R1. Both FICZ, a potent agonist of AHR, and Kyn induced IL-10R1 expression both in vitro and in vivo, and activated an IL-10R1 luciferase promoter construct. Extensions of these studies using loss-of-function strategies revealed that knockdown of either AHR or the AHR binding partner ARNT significantly attenuated FICZ-mediated induction of IL-10R1. AHR was originally identified as the dioxin receptor, regulating genes important for response to various hydrocarbon toxicities. ${ }^{48,49}$ More recent studies have suggested that AHR may have a broader role in the regulation of a range of target genes, including a number of genes important in innate and adaptive immune responses, as well as barrier properties on the skin and gut. ${ }^{50-52}$ In fact, stimulation of AHR with FICZ has been shown to be protective in murine models of colitis. ${ }^{32,43}$ Our finding that IL-10R1 is an AHR target in IEC advances the mechanism(s) of anti-inflammation elicited by AHR activation.

The present findings also demonstrate a prominent role for IEC IL-10R1 expression in wound healing. Our previous findings in vitro and in vivo identified a role for apically 
localized IL-10R1 in the regulation of epithelial barrier function. ${ }^{13}$ Indeed, these studies revealed that shRNAmediated knockdown of IL-10R1 resulted in attenuated barrier formation and decreased baseline barrier function. Similarly, conditional deletion of IL-10R1 in murine IEC resulted in increased intestinal permeability of healthy animals. Extension of these findings here to a scratch wound model revealed a prominent role for the IEC IL-10R in control of wound healing. The combination of AHR ligand and IL-10 significantly enhanced wound closure, thereby adding to our understanding of the protective role of IL-10 during inflammatory processes.

Finally, administration of Kyn during DSS colitis demonstrated a protective role for Kyn increase during intestinal inflammation. Animals that received Kyn not only exhibited fewer physical signs of disease, but there was also significantly less damage to the crypt structure and intestinal barrier function. Kyn is an AHR ligand that can result in many physiological changes to the epithelia/immune cell axis. AHR responses by innate lymphoid cells and intraepithelial lymphocytes have also been shown to be protective during inflammation. Intraepithelial lymphocyte numbers are maintained through AHR stimulation from dietary ligands, ${ }^{53}$ and production of anti-inflammatory cytokines such as IL-22 by innate lymphoid cells in response to AHR ligands has been shown to be protective in colitis. ${ }^{32}$ However, the direct response of the intestinal epithelia to these ligands is less understood. Studies in villin-cre AHR-KO mice confirm that AHR expression within the intestinal epithelia is critical for protection from DSS via Kyn. The increase in IL-10R1 in response to the AHR ligands FICZ and Kyn correlates with the decreased disease scores in Kyn-treated WT mice, whereas the IL-10R1 response to Kyn is abrogated in AHR-KO mice. These results are also in line with the in vitro wound healing assays, demonstrating that increased epithelial IL-10R expression is vital for barrier protection and wound healing in vivo.

Taken together, these findings provide insight into the compelling protective role for AHR in the intestinal epithelium. Cumulatively, the data presented here clarify previous studies suggesting a protective role for Trp metabolism during ongoing inflammatory responses. Similarly, the data presented suggest that IFN $\gamma$, via an IDO1-kyneurinine-AHR link, elicits adaptive mechanisms that promote a program of epithelial wound repair. The significance of these findings in patients is unclear at the present time. It is clear that IDO1 expression is increased in active inflammatory bowel disease and that the serum Kyn:Trp ratio is increased in active Crohn's disease, ${ }^{54}$ suggesting that this metabolic pathway is active during disease. It could be postulated that inflammation-induced increases in IDO1 and the resulting Kyn act as a compensatory mechanism in patients with inflammatory bowel disease.

\section{METHODS}

Metabolomic analysis. Distal colon tissue $(1 \mathrm{~cm})$ and serum were collected from control B6-129 mice and mice with DSS colitis. All tissues were flash frozen and global metabolomics was performed by
Metabolon (Durham, NC). Briefly, colon samples were removed from $-80{ }^{\circ} \mathrm{C}$ and weighed in tared cryovials and $80 \% \mathrm{MetOH}$ was added at a ratio of $75 \mu \mathrm{l}$ solvent per mg of sample, and then incubated overnight at $4{ }^{\circ} \mathrm{C}$ to extract biochemicals. Internal standards were included to control for extraction efficiency. Serum samples were prepared as described previously. ${ }^{55}$ Following extraction in $\mathrm{MetOH}$, both colon and serum samples were processed similarly and analyzed as described. $^{55}$

Animal studies. For the metabolomics analyses, C57B6-129 crossed mice aged 8-12 weeks were given $3 \%\left(\mathrm{wt} \mathrm{vol}^{-1}\right.$ ) DSS (mol. weight 36,000-50,000; MP Biochemicals, Santa Ana, CA) in the drinking water for 5 days. After 5 days, normal tap water was returned for an additional 2 days before tissue collection. Control mice were maintained on tap water for 7 days. The DSS colitis model with Kyn supplementation was performed on C57B6 mice aged 9 weeks as well as 9-week-old AHR villin-cre + and villin-cre- mice with $2.5 \%$ (wt vol ${ }^{-1}$ ) DSS in the drinking water for 6 days. AHR-floxed (AHRfl/ fl) mice containing loxP sites flanking exon 2 of the AHR gene ${ }^{56}$ were crossbred to mice harboring the Cre-recombinase under control of the villin promoter (villin-Cre mice; Jackson Laboratories, Bar Harbor, $\mathrm{ME}$ ). Mice receiving Kyn were injected intraperitoneally with $10 \mathrm{mg} \mathrm{kg}^{-1}$ of Kyn sulfate salt (Sigma, St. Louis, MO) in phosphatebuffered saline (PBS) at day 0 and every $48 \mathrm{~h}$ thereafter. Control mice received PBS injections only. After 6 days, normal tap water was returned for an additional 2 days before tissue collection. Body weight, occult blood in feces, and stool consistency were recorded daily to determine disease activity index as described previously. ${ }^{57}$ ARNTfloxed (ARNTfl/fl) mice containing loxP sites flanking exon 6 of the ARNT gene ${ }^{58}$ (Jackson Laboratories) were cross-bred to mice harboring the Cre-recombinase under control of the villin promoter (villin-Cre mice; Jackson Laboratories). Genotyping of mice was carried out as described previously. Eight- to 12 -week-old female mice were gavaged with $50 \mu \mathrm{g} \mathrm{kg}^{-1}$ FICZ emulsified in $40 \% \mathrm{HP} \beta C D$ ((2-hydroxypropyl)- $\beta$-cyclodextrin) (ArozTech, Cincinnati, OH) or HP $\beta C D$ alone 6 and $12 \mathrm{~h}$ before tissue collection. Cre - littermates were used as controls. Tissues from 24-week-old TNF(deltaARE) mice were harvested to analyze Kyn, IDO1, and IL-10R1. ${ }^{39}$ All animal works were approved by the Institutional Animal Care and Use Committee at the University of Colorado.

Tissue collection, processing, and FITC-dextran permeability Colons with attached ceca were removed and measured. Approximately $1 \mathrm{~cm}$ of whole colon or epithelial scraping was removed and placed in Tris lysis buffer for protein analysis, RNAlater (Ambion, Austin, TX) for qPCR, or flash frozen in liquid nitrogen for HPLC analysis. Isolated epithelial cells were processed using EasySep Mouse Epithelial Enrichment Kit (Stem Cell Technologies, Cambridge, MA). Subsequently, RNA was isolated using RNeasy (Qiagen, Germantown, $\mathrm{MD})$. Four hours before tissue collection, mice were gavaged with $100 \mathrm{mg}$ of $4 \mathrm{kDa}$ FITC-dextran in PBS. Blood for serum analysis was collected via cheek bleeds into serum collection tubes (Sarstedt, Numbrecht, Germany), centrifuged at $5,000 \mathrm{~g}$, and $50 \mu \mathrm{l}$ of serum diluted 1:2 in PBS and placed into wells of a black-walled, clear-bottom 96-well plate. Fluorescence was measured using a Promega Fluorescent Plate Reader (Promega, Madison, WI) at ex. 490 and em. 525, and compared with a standard curve.

Histological scoring. Histological examination was performed on three samples of the distal colon. Samples were fixed in 10\% formalin before staining with hematoxylin and eosin. All histological quantitation was performed in a blinded manner, using a previously described scoring system. ${ }^{59}$ The three independent parameters measured were severity of inflammation ( $0-3$ : none, slight, moderate, severe), extent of injury (0-3: none, mucosal, mucosal and submucosal, transmural), and crypt damage (0-4: none, basal 1/3 damaged, basal $2 / 3$ damaged, only surface epithelium intake, entire crypt and epithelium lost). The score of each parameter was multiplied by a 
factor reflecting the percentage of tissue involvement (x1: 0-25\%; x2: 26-50\%; x3: 51-75\%; x4: 76-100\%) and all numbers were summed. Maximum possible score was 40 .

Cell lines and shRNA knockdown. Human T84 and Caco-2 IECs were cultured in 1:1 Dulbecco's modified Eagle's medium-Ham's F12 with $2.5 \mathrm{~mm}$ L-glutamine and 10\% fetal bovine serum and Dulbecco's modified Eagle's medium with $20 \%$ fetal bovine serum, respectively. Cells were maintained at $37{ }^{\circ} \mathrm{C}$ with $5 \% \mathrm{CO}_{2}$. Lentiviral particles encoding shRNA directed against ARNT, AHR, and IL-10R1 (MISSION TRC shRNA; Sigma) were used to transduce Caco-2 and T84 cells using standard protocols. Stable integration was achieved by puromycin selection $\left(6 \mu \mathrm{g} \mathrm{ml}^{-1}\right)$. Knockdown was confirmed by qPCR analysis, indicating $85 \%$ depletion of both ARNT and AHR levels, and 79\% depletion of IL-10R1. For mRNA and protein analysis, cells were grown to confluence on $5 \mathrm{~cm}^{2}$ permeable supports to form polarized monolayers. Where indicated, cells were treated with the AHR inhibitor CH223191 (Sigma; $10 \mu \mathrm{M}$ ), the IDO1 inhibitor 1-MT (Sigma), recombinant human IFN $\gamma\left(10 \mathrm{ng} \mathrm{ml}^{-1}\right.$; R\&D, Minneapolis, $\mathrm{MN})$, or the AHR agonists FICZ (100 nM-1 $\mu \mathrm{M}$; Enzo, Farmingdale, NY), Kyn (Sigma; $100 \mu \mathrm{m}$ ), or TCDD (Sigma; $30 \mathrm{~nm}$ ) in serum-free media.

EC-HPLC metabolite analysis. Kyn and Trp derivatives were quantified using isocratic reversed-phase high-performance liquid chromatography with EC-HPLC)(Coularray; Thermo Scientific, Waltham, MA). Both tissue and cell samples were extracted in 6\% perchloric acid and protein precipitate and cell debris was removed by centrifugation at $12,000 \mathrm{~g}$. Separation was achieved using an Acclaim Polar Advantage II C18 column, $5 \mu \mathrm{m} 120 \AA$, $4.6 \times 150 \mathrm{~mm}^{2}$ (Thermo Scientific), at a flow rate of $1-1.5 \mathrm{ml} \mathrm{min}^{-1}$ in a mobile phase consisting of $10 \%$ acetonitrile in $50 \mathrm{~mm}$ sodium phosphate buffer, $\mathrm{pH}$ 3 , containing $0.42 \mathrm{~mm}$ octanesulfonic acid as an ion-pairing agent. The data were quantified using the Coularray Software (Waltham, MA) in comparison with standards.

Real-time PCR. Total RNA was extracted from cells using TRIzol (Invitrogen, Carlsbad, CA) and cDNA was prepared using the iSCRIPT cDNA Synthesis Kit (Bio-Rad, Hercules, CA). Real-time PCR to measure IL-10R1 transcript was performed in $1 \times$ Power SYBR Green master mix (Applied Biosystems, Foster City, CA) using an ABI 7300 thermocycler. Fold change in the expression of target mRNA relative to $\beta$-actin mRNA was calculated as outlined previously. ${ }^{60}$ Primer sequences: human IL-10R1, F-5'-CCCTGTCCTATGACCT TACCG- $3^{\prime}$ and $\mathrm{R}-5^{\prime}$-CACACTGCCAACTGTCAGAGT- $3^{\prime}$; human Cyp1A1, F- $5^{\prime}$-ACCCGCCACCCTTCGACAGTTC- $3^{\prime}$ and R-5'-TGC CCAGGCGTTGCGTGAGAAG-3'; human Cyp1B1, F-5' CTGACGACGCCAAGAGACT- $3^{\prime}$ and R-5'-TGGTCTGCTGGAT GGACAGCGGGTT-3'; mouse IL-10R1, F-5'-CCCATTCCTCGTC ACGATC- $3^{\prime}$ and R-5'-TCAGACTGGTTTGGGATAGGTTT- ${ }^{\prime}$; mouse IDO1, $\mathrm{F}-5^{\prime}$-TGGCGTATGTGTGGAACCG-3 ${ }^{\prime}$ and $\mathrm{R}-5^{\prime}-\mathrm{CT}$ CGCAGTAGGGAACAGCAA- $3^{\prime}$; human $\beta$-catenin, $\mathrm{F}-5^{\prime}$-AAAATGG CAGTGCGTTTAG- $3^{\prime}$ and R-5'-TTTGAAGGCAGTCTGTCGTA; human claudin $1, \mathrm{~F}-5^{\prime}$-CCAGTCAATGCCAGGTACGAAT- $3^{\prime}$ and R-5'-TTGGTGTTGGGTAAGAGGTTGTT-3'; human E-cadherin, F- $5^{\prime}$-GCCCATTTCCTAAAAACCTG- $3^{\prime}$ and $\mathrm{R}-5^{\prime}$-CTCTGTCACCT TCAGCCATC-3'; human JAMA, F- $5^{\prime}$-CCTGGGAATCTTGGTTT TTG- $3^{\prime}$ and R-5'-GGAATGACGAGGTCTGTTTG-3'; human occludin, $\mathrm{F}-5^{\prime}$-GCTACGGAAGTGGCTATGG- $3^{\prime}$ and R- $5^{\prime}$-GCGGCAA TGAAACAAAAG- $3^{\prime}$; human ZO-1, F- $5^{\prime}$-TGGTGTCCTACCTAAT TCAACTCA- $3^{\prime}$ and $\mathrm{R}-5^{\prime}$-CGCCAGCTACAAATATTCCAACA- $3^{\prime}$.

Western blot and cell surface ELISA. Whole-cell lysate was extracted in RIPA buffer on ice. Mouse colon samples were collected in Tris lysis buffer and sonicated until homogenous. Protein content was quantified using BCA Protein Assay Reagent (Thermo Scientific, Waltham, MA) and $25 \mu \mathrm{g}$ of whole-cell extract was boiled in Laemmli buffer in reducing conditions and subjected to sodium dodecyl sulfatepolyacrylamide gel electrophoresis. The sodium dodecyl sulfate- polyacrylamide gel electrophoresis was transferred onto PVDF and probed for human IL-10R1 using a rabbit polyclonal IL-10RA antibody (Thermo Scientific), mouse IL-10R1 using a rabbit polyclonal IL-10RA antibody (Millipore, Billerica, MA), mouse IDO1 antibody (Cell Signaling, Danvers, MA), human ARNT antibody (BD Labs, BD Biosciences, San Jose, CA), human AHR antibody (Cell Signaling), and $\beta$-actin (Abcam, Cambridge, MA) according to the manufacturer's recommendations. Densitometry analysis was performed using the ImageJ Software (http://imagej.nih.gov/ij; NIH, Bethesda, MD). Confluent monolayers of Caco-2 cells containing a non-template control (shNTC) or shRNA specific for ARNT were treated with FICZ ( $1 \mu \mathrm{M})$ for $24 \mathrm{~h}$, fixed with $4 \%$ paraformaldehyde, blocked in $10 \%$ bovine serum albumin in PBS, and stained using a rabbit polyclonal IL-10RA antibody (Millipore) at 1:200 overnight at $4{ }^{\circ} \mathrm{C}$. The IL-10RA antibody was detected using an horse radish peroxidase-linked secondary antibody, a TMB peroxidase substrate (KPL, Gaithersberg, $\mathrm{MD})$, and reactivity measured at a wavelength of $450 \mathrm{~nm}$.

Plasmids, transfection, and luciferase assays. The $5^{\prime}$ flanking promoter sequence of the IL-10R1 gene (from -994 to $+23 \mathrm{bp}$ ) was amplified from human genomic DNA and subcloned into the pGL3basic luciferase reporter vector (Promega) to generate pIL-10R1-Luc. Caco- 2 cells were seeded into 24 -well dishes and transfected with the luciferase reporter plasmid pIL-10R1-Luc or empty pGL3-basic-Luc (100 ng) using Lipofectamine-LTX (Invitrogen). To assay promoter activity, cells were exposed to $1 \mu \mathrm{M}$ FICZ, $100 \mu \mathrm{M} \mathrm{Kyn}$, or $10 \mathrm{ng} \mathrm{ml}^{-1}$ IFN $\gamma 24 \mathrm{~h}$ after transfection and harvested at $48 \mathrm{~h}$ after transfection and luciferase activity was measured in extracts using the Dual-Glo Luciferase Reporter Kit and GloMax System (Promega). All firefly luciferase activity was normalized to a co-transfected pRL-SV40 Renilla luciferase reporter (Promega).

Cell proliferation assay. T84 IECs were seeded into 96-well plates at a low density of 5,000 cells per well, allowed to settle, and immediately treated with $250 \mathrm{~nm}$ FICZ, $100 \mu \mathrm{M} \mathrm{Kyn,} 10 \mathrm{ng} \mathrm{ml}^{-1}$ IL-10, vehicle, or a combination. Cell proliferation was measured in relation to dehydrogenase activity as measured by the CCK- 8 assay (Dojindo, Gaithersberg, MD), according to the manufacturer's instructions.

Scratch wound assay. T84 shNTC, shIL-10R1-KD, and shARNT-KD cells were seeded into 6-well dishes and grown to confluence. One centimeter length wounds were scratched into the epithelia using a $10 \mu$ pipette tip and the scratches were monitored daily at $10 \times$ using a Nikon (Melville, NY). Cells were treated daily with fresh media, $250 \mathrm{~nm}$ FICZ, $100 \mu \mathrm{M} \mathrm{Kyn,} \mathrm{and/or} 10 \mathrm{ng} \mathrm{ml}^{-1}$ IL-10. Wound width was measured in three places within the same field over $72 \mathrm{~h}$ using the Spot software (Spot Imaging, Sterling Heights, MI).

Statistical analysis. Data are expressed as the means \pm s.e.m. Statistical analyses were performed using two-tailed unpaired Student's $t$-test in GraphPad Prism (GraphPad, La Jolla, CA) for direct comparisons, one-way analysis of variance with Tukey's test for multiple comparisons, and two-way analysis of variance with Sidak's multiple comparison and multiple $t$-test for grouped data. Statistical significance is reported as ${ }^{\star} P<0.05,{ }^{*} P<0.01$, and ${ }^{* *} P<0.001$.

SUPPLEMENTARY MATERIAL is linked to the online version of the paper at http://www.nature.com/mi

\section{ACKNOWLEDGMENTS}

This work was supported by NIH grants DK099452, DK50189, DK095491, VA Merit BX002182, and by the Crohn's and Colitis Foundation of America.

\section{AUTHOR CONTRIBUTIONS}

J.L. designed and performed experiments, and wrote manuscript. E.A., V.C., D.K., D.K., K.B., and M.G. performed experiments and edited the manuscript. L.G. performed experiments. D.K. and S.C. designed experiments, edited the manuscript, and oversaw the project. 


\section{DISCLOSURE}

The authors declared no conflict of interest.

c 2017 Society for Mucosal Immunology

\section{REFERENCES}

1. Candela, M. et al. Interaction of probiotic Lactobacillus and Bifidobacterium strains with human intestinal epithelial cells: adhesion properties, competition against enteropathogens and modulation of IL-8 production. Int. J. Food Microbiol. 125, 286-292 (2008).

2. Lozupone, C.A., Stombaugh, J.I., Gordon, J.I., Jansson, J.K. \& Knight, R. Diversity, stability and resilience of the human gut microbiota. Nature 489 , 220-230 (2012).

3. Fukuda, S. et al. Bifidobacteria can protect from enteropathogenic infection through production of acetate. Nature 469, 543-547 (2011).

4. Olszak, T. et al. Microbial exposure during early life has persistent effects on natural killer T cell function. Science (New York, NY) 336, 489-493 (2012).

5. Sonnenburg, J.L. et al. Glycan foraging in vivo by an intestine-adapted bacterial symbiont. Science (New York, NY) 307, 1955-1959 (2005).

6. Xavier, R.J. \& Podolsky, D.K. Unravelling the pathogenesis of inflammatory bowel disease. Nature 448, 427-434 (2007).

7. Chaudhry, A. et al. Interleukin-10 signaling in regulatory T cells is required for suppression of Th17 cell-mediated inflammation. Immunity 34, 566-578 (2011).

8. Kuhn, R., Lohler, J., Rennick, D., Rajewsky, K. \& Muller, W. Interleukin-10deficient mice develop chronic enterocolitis. Cell 75, 263-274 (1993).

9. Pils, M.C. et al. Monocytes/macrophages and/or neutrophils are the target of IL-10 in the LPS endotoxemia model. Eur. J. Immunol. 40, 443-448 (2010).

10. Engelhardt, K.R. \& Grimbacher, B. IL-10 in humans: lessons from the gut, IL-10/IL-10 receptor deficiencies, and IL-10 polymorphisms. Curr. Top. Microbiol. Immunol. 380, 1-18 (2014).

11. Glocker, E.O. et al. Inflammatory bowel disease and mutations affecting the interleukin-10 receptor. N. Engl. J. Med. 361, 2033-2045 (2009).

12. Loren, V. et al. Interleukin-10 enhances the intestinal epithelial barrier in the presence of corticosteroids through p38 MAPK activity in Caco-2 Monolayers: a possible mechanism for steroid responsiveness in ulcerative colitis. PLoS One 10, e0130921 (2015).

13. Kominsky, D.J. et al. IFN-gamma-mediated induction of an apical IL-10 receptor on polarized intestinal epithelia. J. Immunol. (Baltimore, MD: 1950) 192, 1267-1276 (2014).

14. Mankertz, J. et al. Expression from the human occludin promoter is affected by tumor necrosis factor alpha and interferon gamma. J. Cell Sci. 113, 2085-2090 (2000).

15. Youakim, A. \& Ahdieh, M. Interferon-gamma decreases barrier function in T84 cells by reducing ZO-1 levels and disrupting apical actin. Am. J. Physiol. 276 (Part 1), G1279-G1288 (1999).

16. Yoshida, R., Imanishi, J., Oku, T., Kishida, T. \& Hayaishi, O. Induction of pulmonary indoleamine 2,3-dioxygenase by interferon. Proc. Natl Acad. SCi. USA 78, 129-132 (1981).

17. Yasui, H., Takai, K., Yoshida, R. \& Hayaishi, O. Interferon enhances tryptophan metabolism by inducing pulmonary indoleamine 2,3-dioxygenase: its possible occurrence in cancer patients. Proc. Natl Acad. Sci. USA 83, 6622-6626 (1986).

18. Higuchi, K. \& Hayaishi, O. Enzymic formation of D-kynurenine from D-tryptophan. Arch. Biochem. Biophys. 120, 397-403 (1967).

19. Munn, D.H., Shafizadeh, E., Attwood, J.T., Bondarev, I., Pashine, A. \& Mellor, A.L. Inhibition of T cell proliferation by macrophage tryptophan catabolism. J. Exp. Med. 189, 1363-1372 (1999).

20. Matteoli, G. et al. Gut CD103 + dendritic cells express indoleamine 2, 3-dioxygenase which influences $\mathrm{T}$ regulatory/T effector cell balance and oral tolerance induction. Gut 59, 595-604 (2010).

21. Nguyen, N.T. et al. Aryl hydrocarbon receptor negatively regulates dendritic cell immunogenicity via a kynurenine-dependent mechanism. Proc. Natl Acad. Sci. USA 107, 19961-19966 (2010).

22. Pallotta, M.T. et al. Indoleamine 2,3-dioxygenase is a signaling protein in long-term tolerance by dendritic cells. Nat. Immunol. 12, 870-878 (2011).
23. Ciorba, M.A. et al. Induction of IDO-1 by immunostimulatory DNA limits severity of experimental colitis. J. Immunol. (Baltimore, MD: 1950) 184, 3907-3916 (2010).

24. Muzaki, A.R. et al. Intestinal CD103CD11b dendritic cells restrain colitis via IFN-gamma-induced anti-inflammatory response in epithelial cells. Mucosal Immunol. 9, 336-351 (2015).

25. Thaker, A.I. et al. IDO1 metabolites activate beta-catenin signaling to promote cancer cell proliferation and colon tumorigenesis in mice. Gastroenterology 145, 416-425 e411-e414 (2013).

26. Opitz, C.A. et al. An endogenous tumour-promoting ligand of the human aryl hydrocarbon receptor. Nature 478, 197-203 (2011).

27. Bessede, A. et al. Aryl hydrocarbon receptor control of a disease tolerance defence pathway. Nature 511, 184-190 (2014).

28. Fernandez-Salguero, P.M., Hilbert, D.M., Rudikoff, S., Ward, J.M. \& Gonzalez, F.J. Aryl-hydrocarbon receptor-deficient mice are resistant to 2,3,7,8-tetrachlorodibenzo-p-dioxin-induced toxicity. Toxicol. Appl. Pharmacol. 140, 173-179 (1996).

29. Gielen, J.E., Goujon, F.M. \& Nebert, D.W. Genetic regulation of aryl hydrocarbon hydroxylase induction. II. Simple Mendelian expression in mouse tissues in vivo. J. Biol. Chem. 247, 1125-1137 (1972).

30. Nguyen, L.P. \& Bradfield, C.A. The search for endogenous activators of the aryl hydrocarbon receptor. Chem. Res. Toxicol. 21, 102-116 (2008).

31. Monteleone, I., MacDonald, T.T., Pallone, F. \& Monteleone, G. The aryl hydrocarbon receptor in inflammatory bowel disease: linking the environment to disease pathogenesis. Curr. Opin. Gastroenterol. 28, 310-313 (2012).

32. Monteleone, I. et al. Aryl hydrocarbon receptor-induced signals upregulate IL-22 production and inhibit inflammation in the gastrointestinal tract. Gastroenterology 141, e231 (2011).

33. Mascanfroni, I.D. et al. Metabolic control of type 1 regulatory $T$ cell differentiation by AHR and HIF1-alpha. Nat. Med. 21, 638-646 (2015).

34. Heller, J.J., Qiu, J. \& Zhou, L. Nuclear receptors take center stage in Th17 cell-mediated autoimmunity. J. Clin. Invest. 121, 519-521 (2011).

35. Kimura, A., Naka, T., Nohara, K., Fujii-Kuriyama, Y. \& Kishimoto, T. Aryl hydrocarbon receptor regulates Stat1 activation and participates in the development of Th17 cells. Proc. Natl Acad. Sci. USA 105, 9721-9726 (2008).

36. Colgan, S.P., Campbell, E.L. \& Kominsky, D.J. Hypoxia and mucosal inflammation. Ann. Rev. Pathol. 11, 77-100 (2016).

37. Taylor, M.W. \& Feng, G.S. Relationship between interferon-gamma, indoleamine 2,3-dioxygenase, and tryptophan catabolism. FASEB J. 5, 2516-2522 (1991).

38. Kominsky, D.J. et al. An endogenously anti-inflammatory role for methylation in mucosal inflammation identified through metabolite profiling. J. Immunol. (Baltimore, MD: 1950) 186, 6505-6514 (2011).

39. Kontoyiannis, D., Pasparakis, M., Pizarro, T.T., Cominelli, F. \& Kollias, G. Impaired on/off regulation of TNF biosynthesis in mice lacking TNFAU-rich elements: implications for joint and gut-associated immunopathologies. Immunity 10, 387-398 (1999).

40. Poland, A., Clover, E., Kende, A.S., DeCamp, M. \& Giandomenico, C.M. $3,4,3^{\prime}, 4^{\prime}$-Tetrachloro azoxybenzene and azobenzene: potent inducers of aryl hydrocarbon hydroxylase. Science (New York, NY) 194, 627-630 (1976).

41. Wincent, E. et al. Inhibition of cytochrome P4501-dependent clearance of the endogenous agonist FICZ as a mechanism for activation of the aryl hydrocarbon receptor. Proc. Natl Acad. Sci. USA 109, 4479-4484 (2012).

42. Bacsi, S.G., Reisz-Porszasz, S. \& Hankinson, O. Orientation of the heterodimeric aryl hydrocarbon (dioxin) receptor complex on its asymmetric DNA recognition sequence. Mol. Pharmacol. 47, 432-438 (1995).

43. Ji, T. et al. Aryl hydrocarbon receptor activation down-regulates IL-7 and reduces inflammation in a mouse model of DSS-induced colitis. Dig. Dis. Sci. 60, 1958-1966 (2015).

44. Kelchtermans, H., Billiau, A. \& Matthys, P. How interferon-gamma keeps autoimmune diseases in check. Trends Immunol. 29, 479-486 (2008).

45. Sheikh, S.Z., Matsuoka, K., Kobayashi, T., Li, F., Rubinas, T. \& Plevy, S.E. Cutting edge: IFN-gamma is a negative regulator of IL-23 in murine macrophages and experimental colitis. J. Immunol. (Baltimore, MD: 1950) 184, 4069-4073 (2010). 
46. Carlin, J.M., Ozaki, Y., Byrne, G.I., Brown, R.R. \& Borden, E.C. Interferons and indoleamine 2,3-dioxygenase: role in antimicrobial and antitumor effects. Experientia 45, 535-541 (1989).

47. Mackenzie, C.R., Langen, R., Takikawa, O. \& Daubener, W. Inhibition of indoleamine 2,3-dioxygenase in human macrophages inhibits interferongamma-induced bacteriostasis but does not abrogate toxoplasmastasis. Eur. J. Immunol. 29, 3254-3261 (1999).

48. Poland, A.P., Glover, E., Robinson, J.R. \& Nebert, D.W. Genetic expression of aryl hydrocarbon hydroxylase activity. Induction of monooxygenase activities and cytochrome P1-450 formation by 2,3,7,8-tetrachlorodibenzo-p-dioxin in mice genetically "nonresponsive" to other aromatic hydrocarbons. J. Biol. Chem. 249, 5599-5606 (1974).

49. Xu, C., Li, C.Y. \& Kong, A.N. Induction of phase I, II and III drug metabolism/ transport by xenobiotics. Arch. Pharm. Res. 28, 249-268 (2005).

50. Fernandez-Salguero, P. et al. Immune system impairment and hepatic fibrosis in mice lacking the dioxin-binding Ah receptor. Science (New York, NY) 268, 722-726 (1995).

51. Esser, C., Rannug, A. \& Stockinger, B. The aryl hydrocarbon receptor in immunity. Trends Immunol. 30, 447-454 (2009).

52. Esser, C. \& Rannug, A. The aryl hydrocarbon receptor in barrier organ physiology, immunology, and toxicology. Pharmacol. Rev. 67, 259-279 (2015).

53. Li, Y. et al. Exogenous stimuli maintain intraepithelial lymphocytes via aryl hydrocarbon receptor activation. Cell 147, 629-640 (2011).
54. Gupta, N.K. et al. Serum analysis of tryptophan catabolism pathway: correlation with Crohn's disease activity. Inflamm. Bowel Dis 18, 1214-1220 (2012).

55. Albrecht, E. et al. Metabolite profiling reveals new insights into the regulation of serum urate in humans. Metabolomics 10, 141-151 (2014).

56. Walisser, J.A., Glover, E., Pande, K., Liss, A.L. \& Bradfield, C.A. Aryl hydrocarbon receptor-dependent liver development and hepatotoxicity are mediated by different cell types. Proc Natl Acad Sci USA 102, 17858-17863 (2005).

57. Frick, J.S., MacManus, C.F., Scully, M., Glover, L.E., Eltzschig, H.K. \& Colgan, S.P. Contribution of adenosine A2B receptors to inflammatory parameters of experimental colitis. J. Immunol. 182, 4957-4964 (2009).

58. Tomita, S., Sinal, C.J., Yim, S.H. \& Gonzalez, F.J. Conditional disruption of the aryl hydrocarbon receptor nuclear translocator (Arnt) gene leads to loss of target gene induction by the aryl hydrocarbon receptor and hypoxia-inducible factor 1alpha. Mol. Endocrinol. 14, 1674-1681 (2000).

59. Dieleman, L.A. et al. Chronic experimental colitis induced by dextran sulphate sodium (DSS) is characterized by Th1 and Th2 cytokines. Clin. Exp. Immunol. 114, 385-391 (1998).

60. Pfaffl, M.W. A new mathematical model for relative quantification in real-time RT-PCR. Nucleic Acids Res. 29, e45 (2001). 